\title{
A Graph Clustering Algorithm Based on Minimum and Normalized Cut
}

\author{
Jiabing Wang ${ }^{1}$, Hong Peng ${ }^{1}$, Jingsong $\mathrm{Hu}^{1}$, and Chuangxin Yang ${ }^{1,2}$ \\ ${ }^{1}$ School of Computer Science and Engineering, South China University of Technology \\ Guangzhou 510641, China \\ ${ }^{2}$ Guangdong University of Commerce, Guangzhou 510320, China \\ \{jbwang, mahpeng, cshjs\} @scut.edu.cn
}

\begin{abstract}
Clustering is the unsupervised classification of patterns into groups. In this paper, a clustering algorithm for weighted similarity graph is proposed based on minimum and normalized cut. The minimum cut is used as the stopping condition of the recursive algorithm, and the normalized cut is used to partition a graph into two subgraphs. The algorithm has the advantage of many existing algorithms: nonparametric clustering method, low polynomial complexity, and the provable properties. The algorithm is applied to image segmentation; the provable properties together with experimental results demonstrate that the algorithm performs well.
\end{abstract}

Keywords: graph clustering, minimum cut, normalized cut, image segmentation.

\section{Introduction}

Clustering is the unsupervised classification of patterns (observations, data items, or feature vectors) into groups (clusters) [1-2]. It groups a set of data in a way that maximizes the similarity within clusters and minimizes the similarity between two different clusters. Due to its wide applicability, the clustering problem has been addressed in many contexts; this reflects its broad appeal and usefulness as one of the steps in exploratory data analysis.

In the past decade, one of the most active research areas of data clustering methods has been spectral graph partition, e.g. [3-7], because of the following advantages: does not need to give the number of clusters beforehand; low polynomial computational complexity, etc. In spectral graph partition, the original clustering problem is first transformed to a graph model; then, the graph is partitioned into subgraphs using a linear algebraic approach.

Minimum cut in similarity graphs were used by $\mathrm{Wu}$ and Leahy [8], Hartuv and Shamir [9]. The minimum cut often causes an unbalanced partition; it may cut a portion of a graph with a small number of vertices [3-4]. In the context of graph clustering, this is, in general, not desirable. To avoid partitioning out a small part of a graph by using edge-cut alone, many graph partitioning criteria were proposed, such as ratio cut [10], normalized cut [4], min-max cut [11], etc. Soundararajan and Sarkar 
[12] have made an in-depth research to evaluate the following partitioning criteria: minimum cut, average cut, and normalized cut.

Normalized cut proposed by Shi and Malik [4] is a spectral clustering algorithm and has been successfully applied to many domains [13-18], especially in image segmentation. However, when applying normalized cut to recursively partitioning data into clusters, a real number parameter - the stopping condition-must be given beforehand [4]. To our knowledge, there are no theoretic results about how to select the parameter. If the parameter is inappropriate, the clustering result is very bad (see an example as shown in Fig. 1 and Fig. 2 in section 2).

In this paper, we propose a clustering algorithm based on minimum and normalized cut. By a novel definition of a cluster for weighted similarity graph, the algorithm does not need to give the stopping condition beforehand and holds many good properties: low polynomial complexity, the provable properties, and automatically determining the number of clusters in the process of clustering.

The rest of the paper is organized as follows. In section 2, we give some basic definitions, a brief review of normalized cut, and the description of our algorithm. In section 3, we prove some properties of the algorithm. In section 4, we apply the algorithm to image segmentation and give some preliminary results. This paper concludes with some comments.

\section{The MAN-C Algorithm}

A weighted, undirected graph $G=(V, E, W)$ consists of a set $V$ of vertexes, a set $E$ of edges, and a weight matrix $W$. The positive weight $w_{i j}$ on an edge connecting two nodes $i$ and $j$ denotes the similarity between $i$ and $j$. For a weighted, undirected graph $G$, we also use $n$ to denote the number of vertexes of $G, m$ the number of edges of $G$, $T$ the sum of weights of all edges of $G$.

The distance $d(u, v)$ between vertices $u$ and $v$ in $G$ is the minimum length of a path joining them, if such path exists; otherwise $d(u, v)=\infty$ (the length of a path is the number of edges in it). The degree of vertex $v$ in a graph, denoted $\operatorname{deg}(v)$, is the number of edges incident on it.

We say that $A$ and $B$ partition the set $V$ if $A \cup B=V$ and $A \cap B=\varnothing$. We denote the partition by the unordered pair $(A, B)$. The cost of a partition for a graph is the sum of the weights of the edges connecting the two parts cut by the partition, i.e.,

$$
\operatorname{Cut}(A, B)=\sum_{i \in A, j \in B} w_{i j} \text {. }
$$

The graph minimum cut (abbreviated min-cut) problem is to find a partition $(A, B)$ such that the cost of the partition is minimal, i.e.,

$$
\min \operatorname{Cut}(A, B)=\min \sum_{i \in A, j \in B} w_{i j} .
$$

Shi and Malik [4] proposed a normalized similarity criterion to evaluate a partition. They call this criterion normalized cut:

$$
N c u t=\frac{\operatorname{Cut}(A, B)}{\operatorname{assoc}(A, V)}+\frac{\operatorname{Cut}(B, A)}{\operatorname{assoc}(B, V)} .
$$


where $\operatorname{assoc}(A, V)=\sum_{i \in A, j \in V} w_{i j}$ is the total connection from nodes in $A$ to all the nodes in the graph, and $\operatorname{assoc}(B, V)$ is similarly defined. It is clear that the optimal partition can be achieved by minimizing Ncut.

The theoretical attraction of the normalized cut lies in its analytical solution. The near-optimal solution of the normalized cut can be obtained by solving the relaxed generalized eigensystem. One key advantage of using normalized cut is that a good approximation to the optimal partition can be computed very efficiently. Let $D$ represent a diagonal matrix such that $D_{i i}=\sum_{j \in V} w_{i j}$, i.e., $D_{i i}$ is the sum of the weights of all the connections to node $i$. Then, the problem of minimizing Ncut can be written as the expression (1), which can be reduced to a generalized eigenvalue system (2) [4].

$$
\begin{gathered}
\text { MinNcut }=\min _{x} \frac{x^{T}(D-W) x}{x^{T} D x} . \\
(D-W) x=\lambda D x .
\end{gathered}
$$

where $x$ represents the eigenvectors, in the real domain, which contain the necessary segmentation information. The eigenvector with the second smallest eigenvalue, called Fiedler vector, is often used to indicate the membership of data points in a subset. Fiedler vector provides the linear search order for the splitting point that can minimize the Ncut objective.

Just as statements in introduction, when applying normalized cut to recursive partitioning data into clusters, a parameter - the stopping condition - must be given beforehand. If the parameter is inappropriate, the clustering result is very bad. An example is given in Fig. 1 and Fig. 2 that show clustering results when given different parameters.

In Fig. 1, the leftmost is the original image, the second one is the clustering result when given the stopping condition $N c u t<0.25$, and the other two images is the
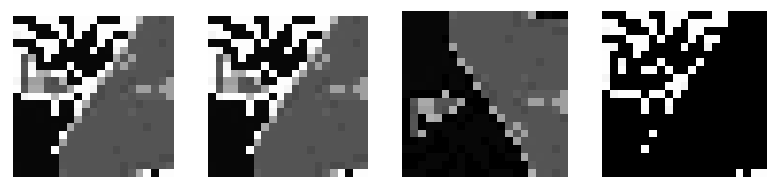

Fig. 1. The leftmost is the original image. The second one is the clustering result when Ncut< 0.25 . The other two images is the clustering result when $N c u t<0.5$.
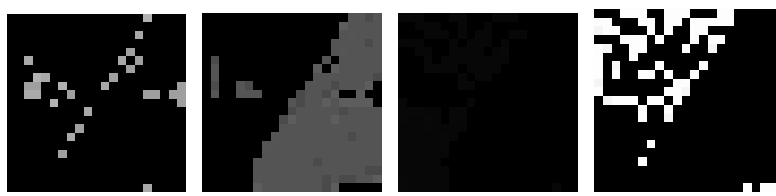

Fig. 2. The clustering result when $N c u t<1$ 
clustering result when given the stopping condition $N c u t<0.5$. Fig. 2 shows the clustering result when given the stopping condition $N c u t<1$. We can see that different parameters result in different clustering results. Especially, when $N c u t<0.25$, the normalized cut cannot segment the original image. So, selection of the stopping condition is very important for applying normalized cut to data clustering. However, to our knowledge, there are no theoretic results on how to select the parameter.

In order to avoid the issue of parameter selection, we give the following definition:

Definition 1. For a graph $G=(V, E, W), G$ is a cluster if and only if $\min$-cut $(G) \geq$ $(n T) /(2 m)$, where $n$ is the number of vertexes of $G, m$ is the number of edges of $G$, and $T$ is the sum of weights of all edges of $G$.

We will see that such a definition of a cluster results in good properties as described in section 3 .

According to the definition 1, we have the clustering algorithm MAN-C (Minimum And Normalized Cut) as shown in Fig. 3.

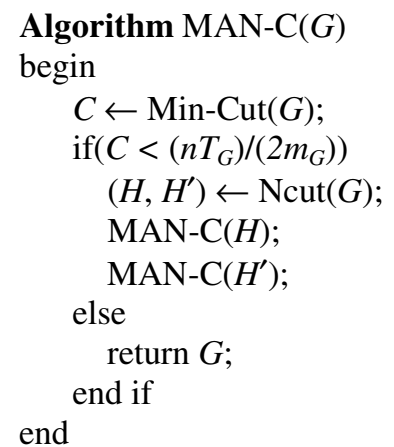

Fig. 3. The MAN-C algorithm

In Fig. 3, $n_{G}$ is the number of vertexes of $G, m_{G}$ is the number of edges of $G$, and $T_{G}$ the sum of weights of all edges of $G$. The procedure $\operatorname{Min}-\operatorname{Cut}(G)$ returns the minimum cut value $C$, and $\operatorname{Ncut}(G)$ returns two subgraphs $H$ and $H^{\prime}$ by implementation of normalized cut. Procedure MAN-C returns a graph in case it identifies it as a cluster, and subgraphs identified as clusters are returned by lower levels of the recursion. Single vertices are not considered clusters and are grouped into a singletons set $S$. The collection of subgraphs returned when applying MAN-C on the original graph constitutes the overall solution.

The running time of the MAN-C algorithm is bounded by $N \times\left(2 f_{l}(n, m)+f_{2}(n)\right)$, where $N$ is the number of clusters found by $\operatorname{MAN}-\mathrm{C}, f_{l}(n, m)$ is the time complexity of computing a minimum cut, and $f_{2}(n, m)$ is the time complexity of computing a normalized cut in a graph with $n$ vertexes and $m$ edges .

The usual approach to solve the min-cut problem is to use its close relationship to the maximum flow problem. Nagamochi and Ibaraki [19] published the first deterministic min-cut algorithm that is not based on a flow algorithm, has the fastest running time of $O(n m)$, but is rather complicated. Stoer and Wagner [20] published a min-cut algorithm with the same running time as Nagamochi and Ibaraki's, but is very simple. 
The normalized cut can be efficiently computed using Lanczos method using the running time $O(k n)+O(k M(n))$ [4], where $k$ is the maximum number of matrix-vector computations required and $M(n)$ is the cost of a matrix-vector computation of $A x$, where $A=D^{-1 / 2}(D-W) D^{-1 / 2}$ (see formula (1)).

\section{Properties of MAN-C Algorithm}

In this section we prove some properties of the clusters produced by the MAN-C algorithm. These demonstrate the homogeneity of the solution.

Definition 2. For a graph $G=(V, E, W)$, a vertex $x \in V$, we define the average edge weight (AEW) as formula (3). That is, the AEW of a vertex $x$ is the average weight of all edges incident on $x$.

$$
\varpi_{x}=\frac{\sum_{v \in V} w_{x v}}{\operatorname{deg}(x)}
$$

Theorem 1. For a cluster $G=(V, E, W)$, the following properties hold:

1. For each pair vertices $v_{1}$ and $v_{2}$, if $\varpi_{v 1} \leq T / m$ and $\varpi_{v 2} \leq T / m$, then the distance between $v_{1}$ and $v_{2}$ is at most two.

2. For each vertex $x \in V, \varpi_{x}>T /(2 m)$.

3. There are $O\left(n^{2}\right)$ edges in $G$.

Proof. Assertion (1) When all edges incident on a vertex are removed, a disconnected graph results. Therefore the following inequality holds according to the definition 1 :

$$
\begin{gathered}
\qquad \sum_{v \in V} w_{x v} \geq \frac{n}{2} \frac{T}{m}, \text { for each } x \in V . \\
\text { equivalently, } \operatorname{deg}(x) \varpi_{x} \geq \frac{n}{2} \frac{T}{m}, \text { for each } x \in V,
\end{gathered}
$$

i.e.,

$$
\operatorname{deg}(x) \geq \frac{n}{2} \frac{T}{m} \frac{1}{\varpi_{x}}, \quad \text { for each } \quad x \in V
$$

So, if $\varpi_{v 1} \leq T / m$ and $\varpi_{v 2} \leq T / m$, then $\operatorname{deg}\left(v_{1}\right) \geq n / 2$, and $\operatorname{deg}\left(v_{2}\right) \geq n / 2$. Since $\operatorname{deg}\left(v_{1}\right)+$ $\operatorname{deg}\left(v_{2}\right) \geq n$, therefore, the distance between $v_{l}$ and $v_{2}$ is at most two, as they have a common neighbor.

Assertion (2) By formula (4), we have:

$$
\varpi_{x} \geq \frac{n}{2 \operatorname{deg}(x)} \frac{T}{m} .
$$

Since $\operatorname{deg}(x) \leq n-1$ for each $x \in V$, we have

$$
\varpi_{x} \geq \frac{n}{2(n-1)} \frac{T}{m}>\frac{T}{2 m} .
$$


Assertion (3) By formula (4), summing over all vertexes in $V$ we get:

$$
\sum_{x \in V} \operatorname{deg}(x) \varpi_{x} \geq n \frac{n}{2} \frac{T}{m}=\frac{n^{2} T}{2 m} .
$$

Equivalently,

$$
2 T \geq \frac{n^{2} T}{2 m} .
$$

That is,

$$
m \geq \frac{n^{2}}{4} .
$$

That is, there are $O\left(n^{2}\right)$ edges in $G$.

The provable properties of MAN-C as shown in Theorem 1 are strong indication of homogeneity. By Theorem 1, the average edge weight of each vertex in a cluster $G$ must be no less than half of average edge weight of $G$, and if the average edge weight of some vertex is small, then it must have more neighbors. Moreover, Theorem 1 shows that each cluster is at least half as dense as a clique, which is another strong indication of homogeneity.

\section{Experimental Results}

Image segmentation is a hot topic in image processing. We have applied MAN-C algorithm to image segmentation. In order to apply the MAN-C to image segmentation, a similarity graph must be constructed. In our experiments, the similarity graph is constructed as follows:

1. Construct a weighted graph $G$ by taking each pixel as a node and connecting each pair of pixels by an edge;

2. For gray image, using just the brightness value of the pixels, we can define the edge weight connecting the two nodes $i$ and $j$ as formula (7);

3. For color image, using HSV values of the pixels, we can define the edge weight connecting the two nodes $i$ and $j$ as formula (8).

$$
w_{i j}=e^{\frac{-(I(i)-I(j))^{2}}{\sigma_{I}}} .
$$

where $I(i)$ is the intensity value for a pixel $i$, and $\sigma_{I}$ is a parameter set to 0.1 [4].

$$
w_{i j}=e^{\frac{-\|F(i)-F(j)\|_{2}^{2}}{\sigma_{I}}}
$$

where $F(i)=[v, v \cdot s \cdot \sin (h), v \cdot s \cdot \cos (h)](i)$, and $h, s, v$ are the HSV values [4].

Considering the space limitation, here we give three examples as shown in Fig. 4, Fig. 5, and Fig. 6. Note that the cluster is drawn using black as background, so the cluster with background is omitted. 

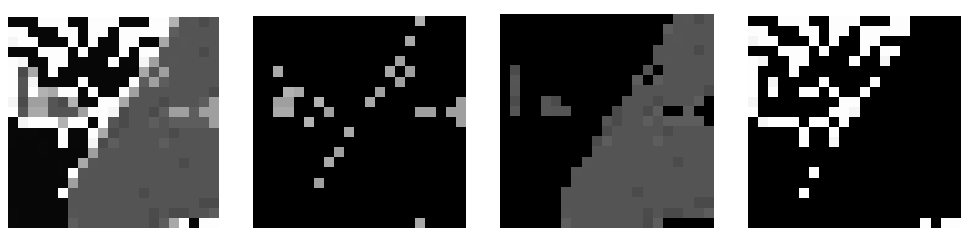

Fig. 4. The leftmost is original image and other images are clusters produced by MAN-C
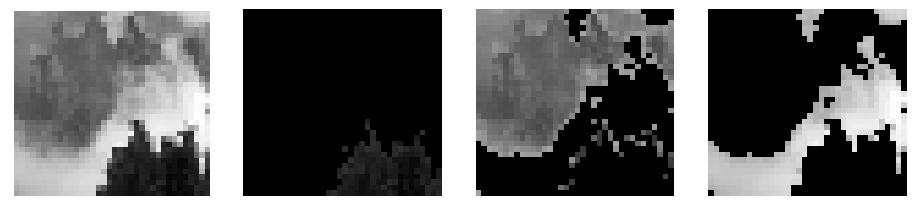

Fig. 5. The leftmost is original image and other images are clusters produced by MAN-C
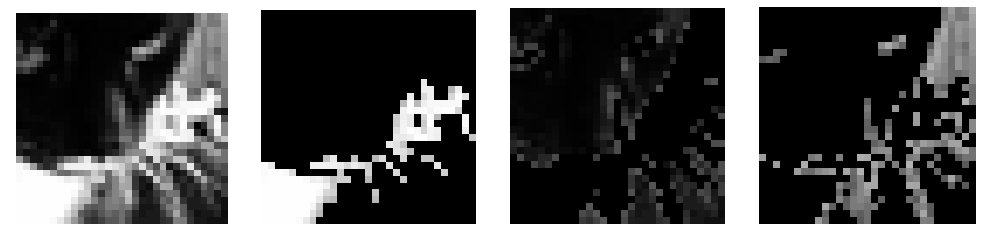

Fig. 6. The leftmost is original image and other images are clusters produced by MAN-C

From the results, we can see that the MAN-C algorithm performs well on all images.

\section{Conclusions}

By a novel definition of a cluster, a graph-theoretic clustering algorithm MAN-C is proposed based on minimum and normalized cut. The minimum cut is used as the stopping condition of the recursive algorithm, and the normalized cut is used to partition a graph into two subgraphs. The MAN-C algorithm has the advantage of many existing algorithm: nonparametric clustering method, low polynomial complexity, and the provable properties. The provable properties of MAN-C together with experimental results demonstrated that the MAN-C algorithm performs well.

\section{Acknowledgements}

This work was supported by Natural Science Foundation of China under Grant No 60574078, Natural Science Foundation of Guangdong Province under Grant No 06300170 and Natural Science Youth Foundation of South China University of Technology under Grant No B07-E5050920. 


\section{References}

1. Jain A.K., Murty M.N., Flynn P.J.: Data Clustering: A Review. ACM Computing Surveys, 31 (1999) 264-323.

2. Xu R., Wunsch II D.: Survey of Clustering Algorithms. IEEE Trans. on Neural Networks, 16(3) (2005) 645-678.

3. Kannan R., Vempala S., Vetta A.: On Clusterings: Good, Bad and Spectral. J. ACM, 51(3) (2004) 497-515.

4. Shi J., Malik J.: Normalized Cuts and Image Segmentation. IEEE Trans. on Pattern Analysis and Machine Intelligence, 22(8) (2000) 888-905.

5. Qiu H., Hancock E. R. Graph Matching and Clustering Using Spectral Partitions. Pattern Recognition, 39 (2006) 22-34.

6. Tritchler D., Fallah S., Beyene J.: A Spectral Clustering Method for Microarray Data. Computational Statistics \& Data Analysis, 49 (2005) 63-76.

7. Van Vaerenbergh S., Santamaría I.: A Spectral Clustering Approach to Underdetermined Postnonlinear Blind Source Separation of Sparse Sources. IEEE Trans. on Neural Networks, 17(3) (2006) 811-814.

8. Wu Z., Leahy R.: An Optimal Graph Theoretic Approach to Data Clustering: Theory and Its Application to Image Segmentation, IEEE Trans. on Pattern Analysis and Machine Intelligence, 15 (11) (1993) 1101-1113.

9. Hartuv E., Shamir R.: A Clustering Algorithm Based on Graph Connectivity. Information Processing Letters, 76 (2000) 175-181.

10. Hagen L., Kahng, A. B.: New Spectral Methods for Ratio Cut Partitioning and Clustering. IEEE Trans. on Computer-Aided Design, 11(9) (1992) 1074-1085.

11. Ding H., He X., Zha H., et al: A Min-Max Cut Algorithm for Graph Partitioning and Data Clustering. In: Proceedings of IEEE 2001 International Conference on Data Mining, IEEE Computer Society Press, Los Almitos, CA, (2001) 107-114.

12. Soundararajan P., Sarkar S.: An In-Depth Study of Graph Partitioning Measures for Perceptual Organization. IEEE Trans. on Pattern Analysis and Machine Intelligence, 25(6) (2003) 642-660.

13. Carballido-Gamio J., Belongie S., Majumdar J. S.: Normalized Cuts in 3-D for Spinal MRI Segmentation. IEEE Trans. on Medical Imaging, 23(1) (2004) 36-44.

14. Duarte A., Sánchez Á., Fernández F., et al: Improving Image Segmentation Quality through Effective Region Merging Using a Hierarchical Social Metaheuristic. Pattern Recognition Letters, 27 (2006) 1239-1251.

15. He X., Zha H., Ding C. H.Q., et al: Web Document Clustering Using Hyperlink Structures. Computational Statistics \& Data Analysis, 41 (2002) 19-45.

16. Li H., Chen W., Shen I-F.: Segmentation of Discrete Vector Fields. IEEE Trans. on Visualization and Computer Graphics, 12(3) (2006) 289-300.

17. Ngo C., Ma Y., Zhang H.: Video Summarization and Scene Detection by Graph Modeling. IEEE Trans. on Circuits and Systems for Video Technology, 15(2) (2005) 296-305.

18. Yu Stella X., Shi J.: Segmentation Given Partial Grouping Constraints. IEEE Trans. on Pattern Analysis and Machine Intelligence, 26(2) (2004) 173-183.

19. Nagamochi H., Ibaraki T.: Computing Edge-Connectivity in Multigraphs and Capacitated Graphs. SIAM J. Discrete Mathematics, 5 (1992) 54-66.

20. Stoer M., Wagner F.: A Simple Min-Cut Algorithm. J. ACM, 44(4) (1997) 585-591. 